\title{
The crossing number of satellite knots
}

\author{
MARC LACKENBY
}

\begin{abstract}
We show that the crossing number of a satellite knot is at least $10^{-13}$ times the crossing number of its companion knot.
\end{abstract}

$57 \mathrm{M} 25$

\section{Introduction}

One of the most basic invariants of a knot $K$ is its crossing number $c(K)$, which is the minimal number of crossings in any of its diagrams. However, it remains quite poorly understood. For example, it is a notorious unsolved conjecture that if $K_{1} \sharp K_{2}$ is the connected sum of two knots $K_{1}$ and $K_{2}$, then $c\left(K_{1} \sharp K_{2}\right)=c\left(K_{1}\right)+c\left(K_{2}\right)$. Connected sums are particular cases of satellite knots, which are defined as follows. Let $L$ be a non-trivial knot in the 3 -sphere. Then a knot $K$ is a satellite knot with companion knot $L$ if $K$ lies in a regular neighbourhood $N(L)$ of $L$, it does not lie in a 3-ball in $N(L)$ and is not a core curve of $N(L)$. (See Figure 1.) It is conjectured that $c(K) \geq c(L)$ (Problem 1.67 in Kirby's problem list [4]). In this paper, we establish that an inequality of this form holds, up to a universally bounded factor.

Theorem 1.1 Let $K$ be a satellite knot with companion knot $L$. Then

$$
c(K) \geq \frac{c(L)}{10^{13}} .
$$

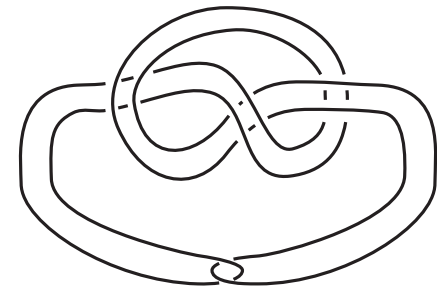

satellite knot

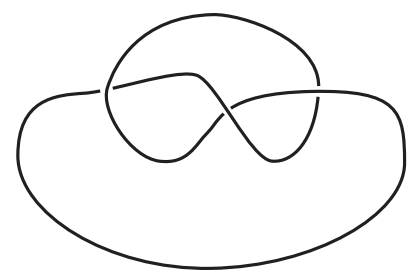

companion knot

Figure 1 
It is possible to formulate stronger conjectures that relate the crossing number of a satellite knot $K$ with that of its companion knot $L$. For example, if $w$ is the wrapping number of the satellite, which is defined to be the minimal number of points of intersection between $K$ and a meridian disc for $N(L)$, then it is conjectured that $c(K) \geq w^{2} c(L)$ (see Hoste, Thistlethwaite and Weeks [3] for instance, where this conjecture is stated explicitly). One may even speculate that there is a formula for $c(K)$ in terms of $w, c(L)$ and the crossing number of $K$ inside $N(L)$ (suitably defined). However, such a formula would need to take account of the writhe of any diagram for $L$, and is unlikely to be neat. In any case, the techniques of this paper will not establish a precise equality of this sort.

Theorem 1.1 should be compared with the main result of the author in [6], which is as follows.

Theorem 1.2 Let $K_{1} \sharp \cdots \sharp K_{n}$ be the connected sum of knots $K_{1}, \ldots, K_{n}$. Then

$$
\frac{c\left(K_{1}\right)+\cdots+c\left(K_{n}\right)}{152} \leq c\left(K_{1} \sharp \cdots \sharp K_{n}\right) \leq c\left(K_{1}\right)+\cdots+c\left(K_{n}\right) .
$$

Both the statement and the proof of Theorem 1.2 will be crucial for us in this paper. Aside from this result, there has not been much work before on the crossing number of satellite knots. In [2], Freedman and He defined the "asymptotic crossing number" of a knot $L$ in terms of the crossing number of certain satellites of $L$. They related this invariant to a physically defined quantity called the "energy" of $L$, and also showed that the asymptotic crossing number is bounded below by a linear function of the genus of $L$. They conjectured that the asymptotic crossing number is equal to the crossing number, a result which would follow from various stronger versions of Kirby's Problem 1.67. It is conceivable that the methods behind the proof of Theorem 1.1 may be applied to obtain new information about the asymptotic crossing number of a knot.

Another key input in the proof of Theorem 1.1 is the machinery developed by the author in [5]. The main goal of that paper was to show that, given any triangulation of the solid torus, there is a core curve (or, more precisely, a "pre-core curve") that lies in the 2-skeleton and that intersects the interior of each face in at most 10 straight arcs. However, in this paper, it is more convenient to use handle structures. In order to be able to speak of "straight" arcs in a handle decomposition, we give it an "affine" structure, which is defined as follows.

Whenever we refer to a handle structure on a 3-manifold, we insist that each handle is attached to handles of strictly lower index. An affine handle structure on a 3-manifold $M$ is a handle structure where each 0 -handle and 1 -handle is identified with a compact 
(but possibly non-convex) polyhedron in $\mathbb{R}^{3}$, so that

(i) each face of each polyhedron is convex,

(ii) whenever a 0 -handle and 1-handle intersect, each component of intersection is identified with a convex polygon in $\mathbb{R}^{2}$, in such a way that the inclusion of this intersection into each handle is an affine map with image equal to a face of the relevant polyhedron,

(iii) for each 0 -handle $H_{0}$, each component of intersection with a 2-handle, 3handle or $\partial M$ is a union of faces of the polyhedron associated with $H_{0}$,

(iv) for each 1-handle, its associated polyhedron is the metric product of a convex 2-dimensional polygon $P$ and an interval $I$, where $P \times \partial I$ is the intersection with the 0 -handles, and the intersection between the 1 -handle and any 2 -handle is $\beta \times I$, where $\beta$ is a union of disjoint sides of $P$.

Since each 0 -handle and 1-handle is identified with a polyhedron, it makes sense to speak of a straight arc in that handle.

The following result of the author was Theorem 4.2 of [5].

Theorem 1.3 Let $\mathcal{H}$ be an affine handle structure of the solid torus $M$. Suppose that each 0 -handle of $\mathcal{H}$ has at most 4 components of intersection with the 1-handles, and that each 1-handle has at most 3 components of intersection with the 2-handles. Then $M$ has a core curve that intersects only the 0 -handles and 1-handles, that respects the product structure on the 1-handles, that intersects each 1-handle in at most 24 straight arcs, and that intersects each 0 -handle in at most 48 arcs. Moreover, the arcs in each 0-handle are simultaneously parallel to a collection of arcs $\alpha$ in the boundary of the corresponding polyhedron, and each component of $\alpha$ intersects each face of the polyhedron in at most 6 straight arcs.

We now give a summary of the proof of Theorem 1.1. It follows a similar route to that of Theorem 1.2, but it requires some new ideas.

We start with a diagram $D$ for the satellite knot $K$ with minimal crossing number. It would suffice to construct a diagram for the companion $L$ with crossing number at most $10^{13} c(D)$. However, instead, we construct a diagram $D^{\prime}$ for a knot $L^{\prime}$, which has $L$ as a connected summand, and with crossing number at most $3 \times 10^{10} c(D)$. Then, applying Theorem 1.2, we deduce that

$$
c(L) \leq 152 c\left(L^{\prime}\right) \leq 152 c\left(D^{\prime}\right) \leq 152 \times 3 \times 10^{10} c(D)<10^{13} c(K),
$$

as required. 
This diagram $D^{\prime}$ is constructed as follows. We use the diagram $D$ to build a handle structure $\mathcal{H}_{X}$ for the exterior $X$ of $K$. Let $T$ be the torus $\partial N(L)$ arising from the satellite construction. Then $T$ is essential in $X$, and so may be placed in normal form with respect to $\mathcal{H}_{X}$. Now cut $X$ along $T$, to give two 3-manifolds, one of which is a copy of the exterior of $L$, the other of which is denoted $Y$ and is $N(L)-\operatorname{int}(N(K))$. The aim is to find a handle structure $\mathcal{H}_{Y^{\prime}}^{\prime}$ for $Y$ that sits nicely in $\mathcal{H}_{X}$. In particular, the 0 -handles (respectively, 1-handles) of $\mathcal{H}_{Y^{\prime}}^{\prime}$ lie in the 0 -handles (respectively, 1-handles) of $\mathcal{H}_{X}$, and each 0 -handle and 1-handle of $\mathcal{H}_{X}$ contains at most 6 such handles of $\mathcal{H}_{Y^{\prime}}^{\prime}$. Now, $Y$ inherits a handle structure $\mathcal{H}_{Y}$ from $\mathcal{H}_{X}$, but it may not have the required properties. This is because the torus $T$ may intersect a handle $H$ of $\mathcal{H}_{X}$ in many normal discs, and these may divide $H$ into many handles of $\mathcal{H}_{Y}$. However, the normal discs come in only finitely many types (at most 5 , in fact, in any handle), and normal discs of the same type are parallel. Between adjacent parallel normal discs, there is an I-bundle, and these patch together to form the parallelity bundle for $\mathcal{H}_{Y}$. This structure was considered in detail by the author in [6], where it was shown that this may be enlarged to another $I$-bundle $\mathcal{B}$ called a generalised parallelity bundle. This has many nice properties. In particular, one may (under certain circumstances) ensure that it consists of $I$-bundles over discs, and other components that have incompressible vertical boundary, which are annuli properly embedded in $Y$, with boundary in $T$. Now, it would be convenient if there were no embedded essential annuli in $Y$ with boundary in $T$, but unfortunately there may be. This happens, for example, if $K$ is also a satellite of a knot $L^{\prime}$ that has $L$ as a connected summand. (An example is shown in Figure 10.) We hypothesise this situation away, by focusing instead on $L^{\prime}$. This is why we aim to find a diagram for $L^{\prime}$ instead of $L$. Using this line of argument, and others, we arrange that $Y$ contains no properly embedded essential annuli with boundary in $T$. Hence, $\mathcal{B}$ consists of $I$-bundles over discs. We replace each of these by a 2 -handle, thereby constructing the required handle structure $\mathcal{H}_{Y^{\prime}}^{\prime}$. We now attach the solid torus $N(K)$, forming a handle structure $\mathcal{H}_{V^{\prime}}$ for the solid torus $N\left(L^{\prime}\right)$. Then, using Theorem 1.3, we find a core curve for $N\left(L^{\prime}\right)$ that lies nicely with respect to $\mathcal{H}_{V^{\prime}}$ and hence $\mathcal{H}_{X}$. This is a copy of $L^{\prime}$, and projecting, we obtain the diagram $D^{\prime}$ for $L^{\prime}$ with the required bound on crossing number.

The factor $10^{13}$ is very large, and one may wonder whether there are ways of reducing it. We have not attempted to optimise this constant. In general, where there was a choice between two arguments, one shorter and simpler than the other, but with worse constants, we have opted for the short and simple route. However, we would be surprised if the constant could be reduced by more than a factor of $10^{8}$ without a major modification to the argument. Nevertheless, there are versions of the theorem with significantly improved constants, but with weakened conclusions. These are as follows. 
Theorem 1.4 Let $K$ be a satellite knot with companion knot $L$. Suppose that there is no essential torus properly embedded in $N(L)-\operatorname{int}(N(K))$. Then

$$
c(K) \geq \frac{c(L)}{3 \times 10^{10}} .
$$

Theorem 1.5 Let $K$ be a satellite knot with companion knot $L$. Suppose that $L$ is prime. Then, for some knot $\tilde{L}$, which is either $L$ or a cable of $L$,

$$
c(K) \geq \frac{c(\tilde{L})}{152} .
$$

The plan of this paper is as follows. In Section 2, we explain how a handle structure on the exterior of a link $K$ can be constructed from any connected diagram for $K$. This is similar to a construction of the author in [6]. However, we then place an affine structure upon this handle decomposition, which is new. In Section 3, we recall some of the normal surface theory that we will need, including the notion of a parallelity bundle and generalised parallelity bundle from [6]. In Section 4, we give the proof of the main theorem, but with one step excluded. It turns out that before the main theorem can be proved in full generality, the special case of certain 2-cables must be analysed separately. We do this in Section 5. Finally, in Section 6, we explain how the proof of the main theorem can be adapted to give Theorem 1.5.

Note that, although our argument uses and extends the techniques developed in [6], we have attempted to make this paper as self-contained as possible.

\section{An affine handle structure from a diagram}

In [6], we introduced a method for creating a handle structure for the exterior of a link $K$, starting from a connected diagram $D$ for $K$. In this section, we give a summary of this construction.

The diagram is a 4 -valent graph embedded in the 2-sphere, and we realise this 2sphere as the equator in $S^{3}$. Let $S^{2} \times[-1,1]$ be a regular neighbourhood of this 2 -sphere, where $S^{2} \times\{0\}$ is the equator itself.

The diagram specifies an embedding of $K$ into the 3-sphere, so that away from small neighbourhoods of the crossings, it lies in the diagram 2-sphere, and at each crossing, two arcs of $K$ come out of the diagram 2-sphere. One goes vertically upwards to height 1 , then runs horizontally, and then returns to the diagram 2 -sphere. The other arc makes a similar itinerary below the diagram. Thus, $K$ lies in $S^{2} \times[-1,1]$ and its 
image under the product projection map to $S^{2}$ equals the 4-valent graph specified by $D$.

The 0 -handles and 1-handles of the handle structure are thickenings of a graph that lies in the equatorial 2-sphere, as follows. There are four 0-handles arranged around each crossing, as in Figure 2. These are joined by four 1-handles that form a square that surrounds the crossing. This square is small enough so that $K$ misses these 1 -handles, because it lies above and below the diagram at these points. In addition, there are two 1-handles that follow each edge of the diagram, and lie either side of that edge. (See Figure 2.)

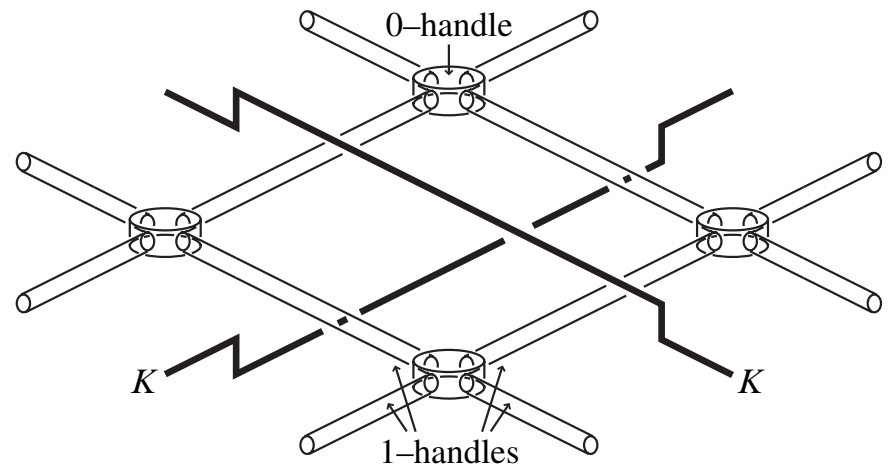

Figure 2

We will not describe in detail here how the 2-handles are attached to the 0 -handles and 1-handles. We merely note that they intersect each 0 -handle as shown in the left of Figure 5.

Finally, there are two 3-handles, one of which lies entirely above the plane of the diagram, and one of which lies below.

We now make a modification to this handle structure. Two new 0 -handles are introduced, which lie either side of an edge of the diagram. The insertion of these handles has the effect of dividing two 1-handles each into two. The two new 0 -handles are known as exceptional. Two new 1-handles are also introduced, each of which joins the two exceptional 0 -handles. These lie above and below the plane of the diagram. They lie in 2-handles of the old handle structure, and so each 1-handle subdivides the 2-handle into two. (See Figure 3.)

We denote the resulting handle structure on the exterior of $K$ by $\mathcal{H}_{X}$.

Note that there is a slight discrepancy between the handle structure here and the one considered by the author in [6]. There, a further modification was made, which removed the two 3-handles, together with two 2-handles. We will not take this step here. 


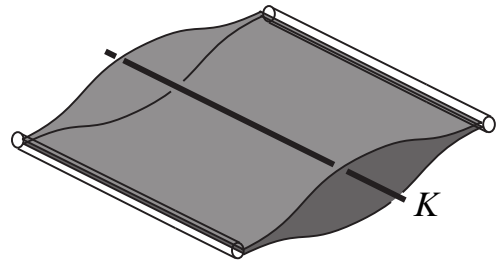

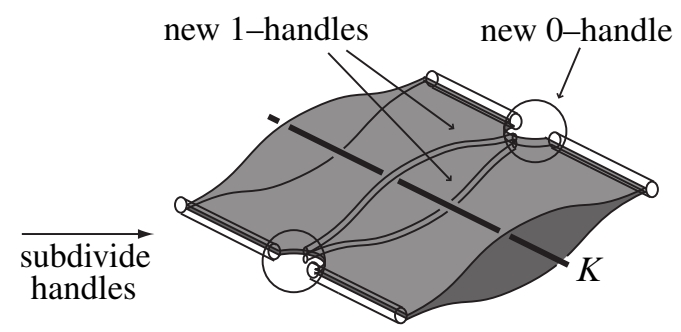

Figure 3

We want to work in $\mathbb{R}^{3}$ rather than $S^{3}$. We therefore pick a point in the diagram 2 -sphere, distant from the crossings, and declare that it is the point at infinity. We thereby obtain a knot diagram in $\mathbb{R}^{2}$ that sits inside $\mathbb{R}^{3}$, and we may assume that the diagrammatic projection map is just the vertical projection from $\mathbb{R}^{3}$ to $\mathbb{R}^{2}$.

We now wish to place an affine structure upon $\mathcal{H}_{X}$. The first step is to realise each 1-handle as a polyhedron. There are two types of 1-handle: those that form part of a square surrounding a crossing, and those that are parallel to an edge of the diagram. We give each of these a slightly different polyhedral structure. Each is the metric product of a convex 2-dimensional polygon and an interval, but the 2-dimensional polygon is a little different in each of the two cases. The precise polygons are shown in Figure 4.

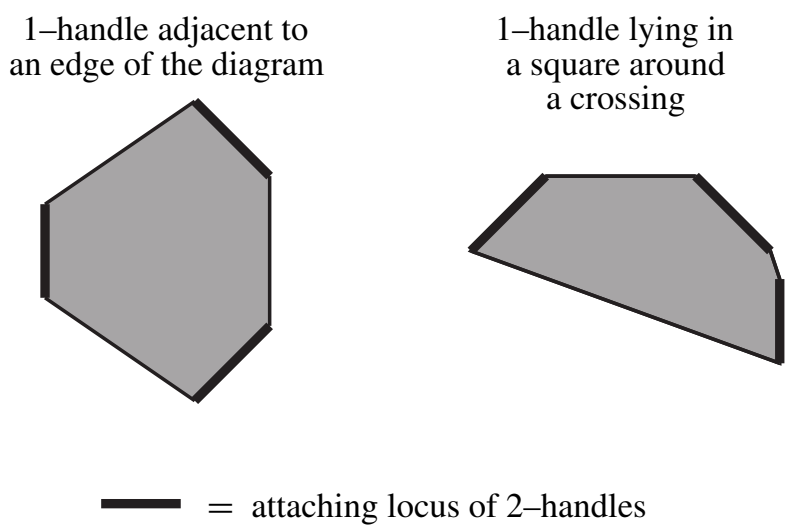

Figure 4

We now realise each 0 -handle as a polyhedron in $\mathbb{R}^{3}$. We focus on the unexceptional 0 -handles. Currently, each is of the form shown in the left of Figure 5. We replace this with the (non-convex) polyhedron shown in the right of Figure 5. Each component of intersection between the 0 -handle and $\partial X$ is realised as a union of 4 triangular faces of the polyhedron (which are not shown in Figure 5). 


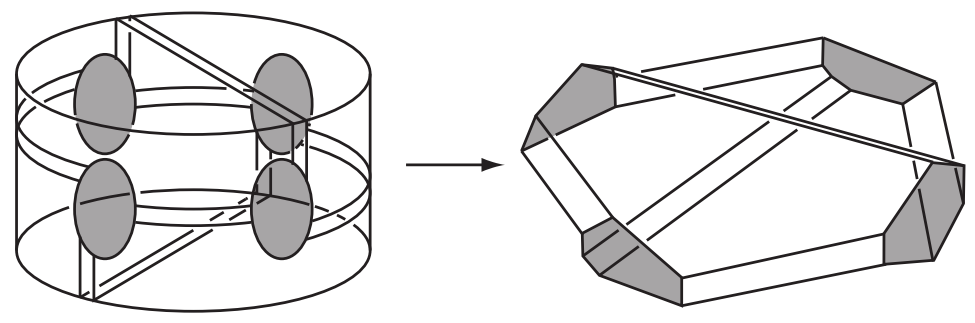

Figure 5

Each polyhedral 0 -handle is embedded in $\mathbb{R}^{3}$ isometrically. But this might not be possible for some of the 1-handles. Some 1-handles follow an edge of the diagram and this edge might not be straight. Nevertheless, there is a way to embed the polyhedron horizontally into $\mathbb{R}^{3}$, so that it has the following property. If the 1 -handle is $D^{1} \times D^{2}$, then for any two distinct points $x_{1}$ and $x_{2}$ in $D^{2}$, the $\operatorname{arcs} D^{1} \times\left\{x_{1}\right\}$ and $D^{1} \times\left\{x_{2}\right\}$ vertically project to arcs in the diagram that either are equal or do not cross.

\section{Generalised parallelity bundles}

As in the proof of Theorem 1.2 that is given in [6], a key technical tool in this paper is the notion of a generalised parallelity bundle. In this section, we will recall this concept, so that the reader does not need to refer to [6]. We will also extend some of the results in [6] so that they can be applied in the context of this paper.

When $\mathcal{H}$ is a handle structure of a 3 -manifold, $\mathcal{H}^{i}$ will denote the union of the $i$-handles.

Convention 3.1 We will insist throughout this paper that any handle structure $\mathcal{H}$ on a 3-manifold satisfies the following conditions:

(i) Each $i$-handle $D^{i} \times D^{3-i}$ intersects $\bigcup_{j \leq i-1} \mathcal{H}^{j}$ in $\partial D^{i} \times D^{3-i}$.

(ii) Any two $i$-handles are disjoint.

(iii) The intersection of any 1-handle $D^{1} \times D^{2}$ with any 2-handle $D^{2} \times D^{1}$ is of the form $D^{1} \times \alpha$ in $D^{1} \times D^{2}$, where $\alpha$ is a collection of arcs in $\partial D^{2}$, and of the form $\beta \times D^{1}$ in $D^{2} \times D^{1}$, where $\beta$ is a collection of arcs in $\partial D^{2}$.

(iv) Each 2-handle of $\mathcal{H}$ runs over at least one 1-handle. 
The handle structure constructed in Section 2 satisfies these requirements.

Now let $X$ be a compact orientable 3-manifold with a handle structure $\mathcal{H}_{X}$.

Let $\mathcal{F}$ be the surface $\mathcal{H}_{X}^{0} \cap\left(\mathcal{H}_{X}^{1} \cup \mathcal{H}_{X}^{2}\right)$, let $\mathcal{F}^{0}$ be $\mathcal{H}_{X}^{0} \cap \mathcal{H}_{X}^{1}$, and let $\mathcal{F}^{1}$ be $\mathcal{H}_{X}^{0} \cap \mathcal{H}_{X}^{2}$. By the above conditions, $\mathcal{F}$ is a thickened graph, where the thickened vertices are $\mathcal{F}^{0}$ and the thickened edges are $\mathcal{F}^{1}$.

Definition 3.2 We say that a surface $T$ properly embedded in $X$ is standard if:

(i) It intersects each 0 -handle in a collection of properly embedded disjoint discs.

(ii) It intersects each 1 -handle $D^{1} \times D^{2}$ in $D^{1} \times \beta$, where $\beta$ is a collection of properly embedded disjoint arcs in $D^{2}$.

(iii) It intersects each 2-handle $D^{2} \times D^{1}$ in $D^{2} \times P$, where $P$ is a collection of points in the interior of $D^{1}$.

(iv) It is disjoint from the 3-handles.

See Figure 6.

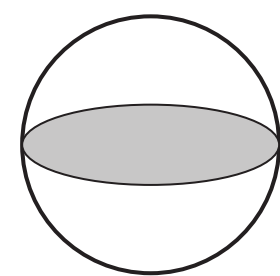

0-handle

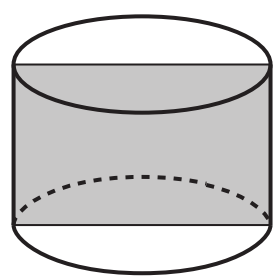

1-handle

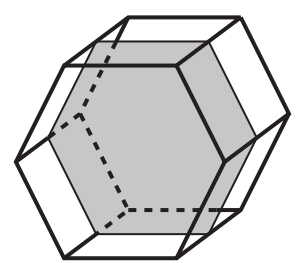

2-handle

Figure 6

Definition 3.3 A disc component $D$ of $T \cap \mathcal{H}_{X}^{0}$ is said to be normal if:

(i) $\partial D$ intersects any thickened edge of $\mathcal{F}$ in at most one arc.

(ii) $\partial D$ intersects any component of $\partial \mathcal{F}^{0}-\mathcal{F}^{1}$ at most once.

(iii) $\partial D$ intersects each component of $\partial \mathcal{H}^{0}-\mathcal{F}$ in at most one arc and no simple closed curves.

A standard surface that intersects each 0 -handle in a disjoint union of normal discs is said to be normal. (See Figure 7.) 
These cannot be part of the same normal disc $D$, by (i)

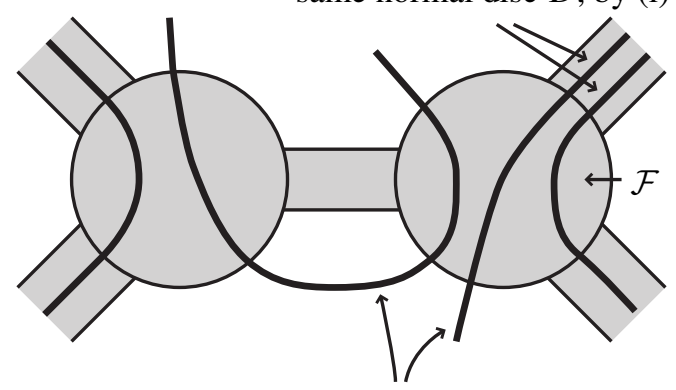

These cannot be part of the same normal disc $D$, by (ii) or (iii)

Figure 7

This is a slightly weaker definition of normality than is used by some authors, for example by Matveev in [7, Definition 3.4.1]. However, if we had used the definition in [7], Proposition 3.4 (below) would no longer have held true.

When $T$ has no boundary and $H_{0}$ is a 0 -handle such that $H_{0} \cap \mathcal{F}$ is a thickening of the complete graph on 4 vertices, the above conditions imply that $H_{0} \cap T$ is a collection of triangles and squares, as shown in Figure 8.

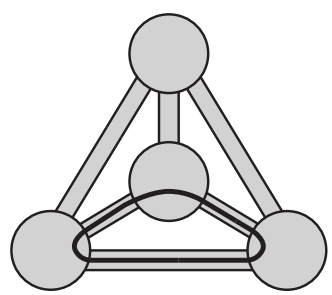

Triangle

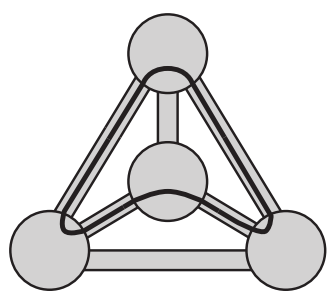

Square

Figure 8

We say that a simple closed curve properly embedded in $\partial X$ is standard if:

(i) It is disjoint from the 2-handles.

(ii) It intersects each 1 -handle $D^{1} \times D^{2}$ in $D^{1} \times P$, where $P$ is a finite set of points in $\partial D^{2}$.

(iii) It intersects $\operatorname{cl}\left(\partial \mathcal{H}_{X}^{0}-\mathcal{F}\right)$ in a collection of properly embedded arcs.

The following is Proposition 4.4 in [6]. It is a variant of a well-known result in normal surface theory. 
Proposition 3.4 Let $\mathcal{H}_{X}$ be a handle structure on a compact irreducible 3-manifold $X$. Let $T$ be a properly embedded, incompressible, boundary-incompressible surface in $X$, with no 2-sphere components. Suppose that each component of $\partial T$ is standard and intersects each component of $\partial X \cap \mathcal{H}_{X}^{0}$ and $\partial X \cap \mathcal{H}_{X}^{1}$ in at most one arc and no simple closed curves. Then there is an ambient isotopy, supported in the interior of $X$, taking $T$ into normal form.

In the remainder of this section, $M$ will always be a compact orientable 3-manifold with a handle structure $\mathcal{H}_{M}$, and $S$ will be a compact subsurface of $\partial M$ such that $\partial S$ is standard. In this case, we say that $\mathcal{H}_{M}$ is a handle structure for the pair $(M, S)$.

Definition 3.5 Let $\mathcal{H}_{M}$ be a handle structure for the pair $(M, S)$. A handle $H$ of $\mathcal{H}_{M}$ is a parallelity handle if it admits a product structure $D^{2} \times I$ such that:

(i) $D^{2} \times \partial I=H \cap S$

(ii) Each component of $\mathcal{F}^{0} \cap H$ and $\mathcal{F}^{1} \cap H$ is $\beta \times I$, for a subset $\beta$ of $\partial D^{2}$.

We will typically view the product structure $D^{2} \times I$ as an $I$-bundle over $D^{2}$.

The main example of a parallelity handle arises when $M$ is obtained by cutting a 3-manifold $X$ along a properly embedded, normal surface $T$, and where $S$ is the copies of $T$ in $M$. Then, if $T$ contains two normal discs in a handle that are normally parallel and adjacent, the space between them becomes a parallelity handle in $(M, S)$.

The $I$-bundle structures on the parallelity handles can be chosen so that, when two parallelity handles are incident, their $I$-bundle structures coincide along their intersection. (This is established in the proof of Lemma 5.3 in [6].) So, the union of the parallelity handles forms an $I$-bundle over a surface $F$. This is termed the parallelity bundle $\mathcal{B}$. The $I$-bundle over $\partial F$ is termed the vertical boundary $\partial_{v} \mathcal{B}$ of $\mathcal{B}$, and the $\partial I$-bundle over $F$ is called the horizontal boundary $\partial_{h} \mathcal{B}$.

As in [6], it will be technically convenient to consider enlargements of such structures. These will still be an $I$-bundle over a surface $F$, and near the $I$-bundle over $\partial F$, they will be a union of parallelity handles, but elsewhere need not be. The precise definition is as follows.

Definition 3.6 Let $\mathcal{H}_{M}$ be a handle structure for the pair $(M, S)$. A generalised parallelity bundle $\mathcal{B}$ is a 3 -dimensional submanifold of $M$ such that:

(i) $\mathcal{B}$ is an $I$-bundle over a compact surface $F$.

(ii) The $\partial I$-bundle is $\mathcal{B} \cap S$. 
(iii) $\mathcal{B}$ is a union of handles of $\mathcal{H}_{M}$.

(iv) Any handle in $\mathcal{B}$ that intersects the $I$-bundle over $\partial F$ is a parallelity handle, where the $I$-bundle structure on the parallelity handle agrees with the $I$-bundle structure of $\mathcal{B}$.

(v) The restriction of $\mathcal{H}_{M}$ to $\operatorname{cl}(M-\mathcal{B})$ is a handle structure. (In the presence of the other conditions, this is equivalent to the requirement that whenever an $i$-handle lies in $\mathcal{B}$, then so do all the $j$-handles that are attached to it, where $j>i$.)

The $I$-bundle over $\partial F$ is termed the vertical boundary $\partial_{v} \mathcal{B}$ of $\mathcal{B}$, and the $\partial I$-bundle over $F$ is called the horizontal boundary $\partial_{h} \mathcal{B}$.

We will also need to make some modifications to handle structures, as follows.

Definition 3.7 Let $G$ be an annulus properly embedded in $M$, with boundary in $S$. Suppose that there is an annulus $G^{\prime}$ in $\partial M$ such that $\partial G=\partial G^{\prime}$. Suppose also that $G \cup G^{\prime}$ bounds a $3-$ manifold $P$ such that:

(i) Either $P$ is a parallelity region between $G$ and $G^{\prime}$, or $P$ lies in a 3-ball $B$ such that $B \cap \partial M$ is a disc.

(ii) $P$ is a non-empty union of handles.

(iii) The restriction of $\mathcal{H}_{M}$ to $\operatorname{cl}(M-P)$ is a handle structure.

(iv) Any parallelity handle of $\mathcal{H}_{M}$ that intersects $P$ lies in $P$.

(v) $G$ is a vertical boundary component of a generalised parallelity bundle lying in $P$.

Removing the interiors of $P$ and $G^{\prime}$ from $M$ is called an annular simplification. Note that the resulting 3-manifold $M^{\prime}$ is homeomorphic to $M$, even though $P$ may be homeomorphic to the exterior of a non-trivial knot when it lies in a 3-ball. (See Figure 9.) The boundary of $M^{\prime}$ inherits a copy of $S$, which we denote by $S^{\prime}$, by setting $S^{\prime}=\left(S \cap \partial M^{\prime}\right) \cup\left(\partial M^{\prime}-\partial M\right)$. Thus, $\left(M^{\prime}, S^{\prime}\right)$ is homeomorphic to $(M, S)$. Moreover, when $M$ is embedded within a bigger closed 3-manifold, then $\left(M^{\prime}, S^{\prime}\right)$ is ambient isotopic to $(M, S)$.

This definition is similar to that given by the author in [6]. However, there, $S$ was required to be incompressible. We do not make that assumption here, but as a result, condition (i) has been modified a little.

The following result of the author is Lemma 5.5 in [6]. 


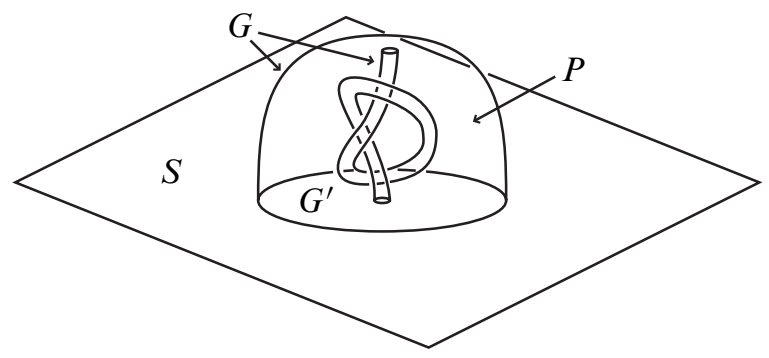

Figure 9

Lemma 3.8 Let $\mathcal{H}_{M}$ be a handle structure for the pair $(M, S)$. Let $\mathcal{H}_{M}^{\prime}$ be a handle structure obtained from $\mathcal{H}_{M}$ by annular simplifications. Then any parallelity handle for $\mathcal{H}_{M}$ that lies in $\mathcal{H}_{M}^{\prime}$ is a parallelity handle for $\mathcal{H}_{M}^{\prime}$.

The following is Corollary 5.7 in [6].

Theorem 3.9 Let $M$ be a compact orientable irreducible 3-manifold with a handle structure $\mathcal{H}_{M}$. Let $S$ be an incompressible subsurface of $\partial M$ such that $\partial S$ is standard in $\partial M$. Suppose that $\mathcal{H}_{M}$ admits no annular simplification. Then $\mathcal{H}_{M}$ contains a generalised parallelity bundle that contains every parallelity handle and that has incompressible horizontal boundary.

From this, we deduce the following.

Theorem 3.10 Let $M$ be a compact connected orientable irreducible 3-manifold, with boundary a collection of incompressible tori. Let $S$ be a union of components of $\partial M$. Let $\mathcal{H}_{M}$ be a handle structure for $(M, S)$ that admits no annular simplification. Suppose that there is no essential properly embedded annulus in $M$, with boundary in $S$, and that lies entirely in the parallelity handles. Suppose also that $M$ is not an $I-$ bundle over a torus or Klein bottle. Then $M$ contains a generalised parallelity bundle $\mathcal{B}$ such that:

(i) $\mathcal{B}$ contains every parallelity handle.

(ii) $\mathcal{B}$ is a collection of $I-$ bundles over discs.

This is very close to the statement of Proposition 5.8 in [6]. However, there, $S$ was a collection of annuli, whereas here it is tori. In addition, a slightly more precise hypothesis has been made about the annuli properly embedded in $M$. 
Proof By Theorem 3.9, there is a generalised parallelity bundle $\mathcal{B}$ that contains every parallelity handle and with incompressible horizontal boundary. Let $\mathcal{B}^{\prime}$ be the union of the components of $\mathcal{B}$ that are not $I$-bundles over discs. Its horizontal boundary $\partial_{h} \mathcal{B}^{\prime}$ is a subsurface of $S$, and hence a collection of annuli and tori. However, if there is any toral component of $\partial_{h} \mathcal{B}^{\prime}$, then this lies in a component of $\mathcal{B}^{\prime}$ that is an $I$-bundle over a Klein bottle or torus. This then is all of $M$, which is contrary to hypothesis. Hence, the horizontal boundary of $\mathcal{B}^{\prime}$ just consists of annuli. By hypothesis, each component of $\partial_{v} \mathcal{B}^{\prime}$ is inessential. But $\partial_{v} \mathcal{B}^{\prime}$ is incompressible, and so each component of $\partial_{v} \mathcal{B}^{\prime}$ is boundary parallel.

Since the horizontal boundary of $\mathcal{B}^{\prime}$ consists of annuli, we deduce that $\mathcal{B}^{\prime}$ is a collection of $I$-bundles over Möbius bands and annuli. We now show that, in fact, no component of $\mathcal{B}^{\prime}$ is an $I$-bundle over a Möbius band. Let $B$ be such a component, and let $A$ be its vertical boundary, which is an annulus. We know that $A$ is boundary-parallel, via a parallelity region $P$, say. Now the interior of $P$ is disjoint from $B$, since $B$ is an $I$-bundle over a Möbius band. Hence, $P \cup B$ is all of $M$, and we deduce that $M$ is a solid torus, which is contrary to assumption. This proves the claim.

So, each component of $\mathcal{B}^{\prime}$ is an $I$-bundle over an annulus. Let $B$ be such a component, and let $A$ be one its vertical boundary components. This is boundary-parallel, via a parallelity region $P$. By choosing $A$ appropriately, we may assume that $B$ lies in $P$. Therefore, removing $P-A$ from $\mathcal{H}_{M}$ is an annular simplification. But this contradicts the assumption that $\mathcal{H}_{M}$ admits no annular simplifications. We therefore deduce that $\mathcal{B}^{\prime}$ is empty, and hence that $\mathcal{B}$ is a collection of $I$-bundles over discs.

\section{Proof of the main theorem}

Let $K$ be a satellite knot with companion knot $L$. Let $X$ be the exterior of $K$.

Claim There is a companion knot $L^{\prime}$ for $K$ with the following properties:

(i) $L^{\prime}$ has $L$ as a connected summand (possibly $L^{\prime}=L$ ).

(ii) $L^{\prime}$ is not a non-trivial connected summand for any other companion for $K$.

Suppose that $L$ fails condition (ii). In other words, suppose that there is another companion $L_{1}$ for $K$, such that $L_{1}$ is the connected sum of $L$ and some other nontrivial knot. Then $L_{1}$ is a satellite of $L$, and so we have an inclusion $K \subset N\left(L_{1}\right) \subset$ $N(L)$. (See Figure 10.) This knot $L_{1}$ may also fail condition (ii), but in this case we get another knot $L_{2}$ such that $K \subset N\left(L_{2}\right) \subset N\left(L_{1}\right) \subset N(L)$, and where $L_{2}$ has $L$ as a connected summand, and so on. Each torus $\partial N\left(L_{i}\right)$ is essential in $X$, and they 

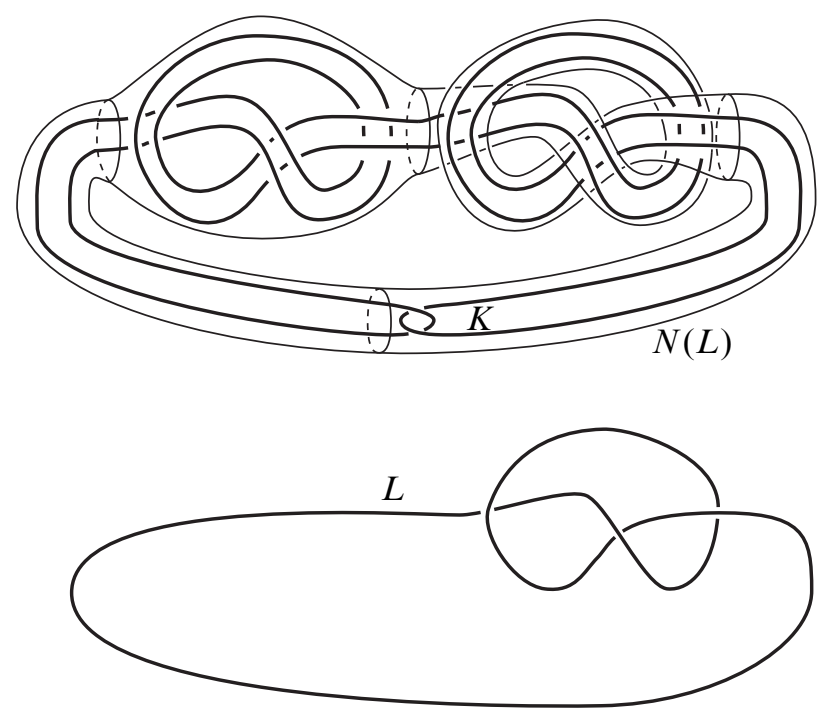

Figure 10

are disjoint and non-parallel. By Kneser's theorem, there is an upper bound on the number of such tori. Hence, eventually, we obtain the required knot $L^{\prime}$, as claimed.

Let $D$ be a diagram of $K$ with minimal crossing number. Our aim is to construct a diagram $D^{\prime}$ for $L^{\prime}$ with crossing number at most $3 \times 10^{10} c(D)$. Then, applying Theorem 1.2, we deduce that

$$
c(L) \leq 152 c\left(L^{\prime}\right) \leq 152 c\left(D^{\prime}\right) \leq 152 \times 3 \times 10^{10} c(D)<10^{13} c(K),
$$

thereby proving Theorem 1.1. This will also prove Theorem 1.4, because if there is no essential torus in $N(L)-\operatorname{int}(N(K))$, then we must have $L^{\prime}=L$.

We may assume that $K$ does not have $L^{\prime}$ as a connected summand, because by Theorem 1.2 , this would imply that $c(K) \geq c\left(L^{\prime}\right) / 152$, which is stronger than the required inequality.

Give $X$ the handle structure described in Section 2, which we denote by $\mathcal{H}_{X}$. Let $V$ be the solid torus $N\left(L^{\prime}\right)$, and let $T$ be the torus $\partial V$. Since $T$ is incompressible in $X$, it may be placed in normal form with respect to $\mathcal{H}_{X}$. Note that $T$ then inherits a handle structure where the $i$-handles are $T \cap \mathcal{H}_{X}^{i}$. Cutting $X$ along $T$ gives two 3-manifolds, one of which is a copy of the exterior of $L^{\prime}$, the other of which is $V$ with the interior of a small regular neighbourhood of $K$ removed. Let $Y$ be this latter manifold. It inherits a handle structure $\mathcal{H}_{Y}$. 
We wish to place an affine structure upon $\mathcal{H}_{Y}$. To do this, we start by straightening $T$ as much as possible, in the following way. In each 1 -handle $D^{1} \times D^{2}$ of $\mathcal{H}_{X}$, we realise $T \cap\left(D^{1} \times D^{2}\right)$ as $D^{1} \times \alpha$, where $\alpha$ is a collection of straight arcs in the polygonal structure on $D^{2}$. Then we make each arc of $T \cap \mathcal{H}_{X}^{0} \cap \mathcal{H}_{X}^{2}$ straight. Thus the boundary of each normal disc of $T$ in each 0 -handle is now a concatenation of straight arcs. We then realise each normal disc of $T$ in $\mathcal{H}_{X}^{0}$ as a union of flat polygons in that 0 -handle, as follows.

Observe first that although the polyhedron $P$ associated with each 0 -handle is not convex, it is star-shaped. In other words, there is a point $v$ in the interior of $P$, such that for each point on $\partial P$, the straight line joining it to $v$ lies within $P$. Moreover, the interior of this straight line lies within the interior of $P$. We may therefore use a dilation about $v$, with any positive scale factor less than 1 , to create a copy of $\partial P$ lying within the interior of $P$. Create a nested collection of such copies of $\partial P$, the number of copies being equal to the number of normal discs that we need to insert into $P$. We now create these normal discs one at a time, starting with one that has boundary that is innermost in $\partial P$. The boundary of this disc has already been specified as some curve $C$ in $\partial P$. Use the star-shaped nature of $P$ to create an annulus interpolating between $C$ and a curve on the outermost copy of $\partial P$. Since $C$ is a union of straight arcs, this annulus is a union of flat quadrilaterals. The boundary component of this annulus that lies on the dilated copy of $\partial P$ bounds a disc in this copy of $\partial P$ that is a union of flat polygons. The union of these with the annulus is the required normal disc bounded by $C$. We now repeat this procedure for the remaining curves of $T \cap \partial P$, starting with the next innermost curve, and using the next copy of $\partial P$. In this way, each normal disc of $T$ has been realised as a union of flat polygons.

We now want to bound the number of flat polygons that comprise each normal disc. The boundary of each normal triangle is 6 straight arcs, and these give rise to 6 flat polygons in the annular part of the normal disc, together with 10 flat polygons in the copy of $\partial P$. This is 16 in total. Similarly, one can compute that each normal square is composed of 25 flat polygons.

Having realised $T$ in this way, we cut $\mathcal{H}_{X}$ along $T$, and then $\mathcal{H}_{Y}$ inherits an affine handle structure. We view this as a handle structure for the pair $(Y, T)$. Thus, whenever we consider a parallelity handle in $\mathcal{H}_{Y}$, its horizontal boundary lies in the copy of $T$ in $Y$. (See Figure 11.)

Each 0 -handle $H_{0}$ of $\mathcal{H}_{X}$ gives rise to at most 6 handles of $\mathcal{H}_{Y}$ that are not parallelity handles. (This number 6 arises when we cut the 0 -handle along 4 normal triangles of distinct types and a normal square.) Let $H_{0}^{\prime}$ be the non-parallelity 0 -handles of $\mathcal{H}_{Y}^{0}$ lying in $H_{0}$. We wish to bound the number of faces in the polyhedral structure of $H_{0}^{\prime}$. 


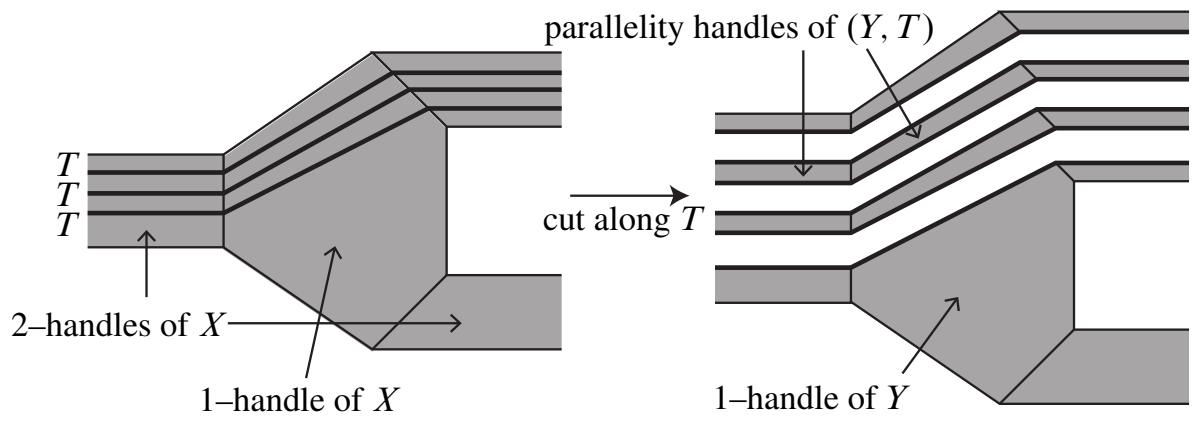

Figure 11

Clearly, the maximal number of faces occurs when $H_{0}$ is an unexceptional 0 -handle that is cut along all 4 triangle types and a square type, and so it is this configuration that we will examine.

The faces of $H_{0}$ come in the following types: the intersections with $\mathcal{H}_{X}^{1}$ (of which there are 4 ), the intersections with $\mathcal{H}_{X}^{2}$ (of which there are 6) and the intersections with $\partial X$ (of which there are 4 components, forming a total of 16 faces). Each face in $H_{0} \cap \mathcal{H}_{X}^{1}$ gives rise to 5 faces of $H_{0}^{\prime} \cap \mathcal{H}_{Y}^{1}$. The faces of $H_{0} \cap \mathcal{H}_{X}^{2}$ become 22 faces of $H_{0}^{\prime} \cap \mathcal{H}_{Y}^{2}$. The faces of $H_{0} \cap \partial X$ stay as faces of $H_{0}^{\prime}$. Finally, the normal triangles and squares of $T$ give rise to $(16 \times 8)+(25 \times 2)=178$ faces of $H_{0}^{\prime}$. So, in total, the number of faces of $H_{0}^{\prime}$ is at most $(4 \times 5)+22+(4 \times 4)+178=236$.

Apply as many annular simplifications to $\mathcal{H}_{Y}$ as possible, creating a handle structure $\mathcal{H}_{Y^{\prime}}$ for an isotopic copy of $(Y, T)$, which we call $\left(Y^{\prime}, T^{\prime}\right)$. By Theorem 3.9, $\mathcal{H}_{Y^{\prime}}$ contains a generalised parallelity bundle $\mathcal{B}$ that contains every parallelity handle of $\mathcal{H}_{Y^{\prime}}$, and that has incompressible horizontal boundary. Note that $\mathcal{H}_{Y^{\prime}}$ inherits an affine handle structure, since it is a union of handles of $\mathcal{H}_{Y}$.

Since $\partial_{h} \mathcal{B}$ is an incompressible subsurface of $T^{\prime}$, it is a collection of discs and annuli. Thus, $\mathcal{B}$ is a collection of $I$-bundles over discs, annuli and Möbius bands.

The proof now divides into two cases.

Case 1 Some component of $\mathcal{B}$ is an $I$-bundle over a Möbius band.

This component of $\mathcal{B}$ is a solid torus, and its horizontal boundary is an annulus that winds twice around the solid torus $Y^{\prime} \cup N(K)$. Each boundary component of this annulus is therefore a 2-cable of $L^{\prime}$. Recall that a knot $C$ is a 2 -cable of a knot $L^{\prime}$ if $C$ is the boundary of an embedded Möbius band, the core of which is a copy of $L^{\prime}$. The linking number between $C$ and this core curve is the twisting number of the 2-cable. 


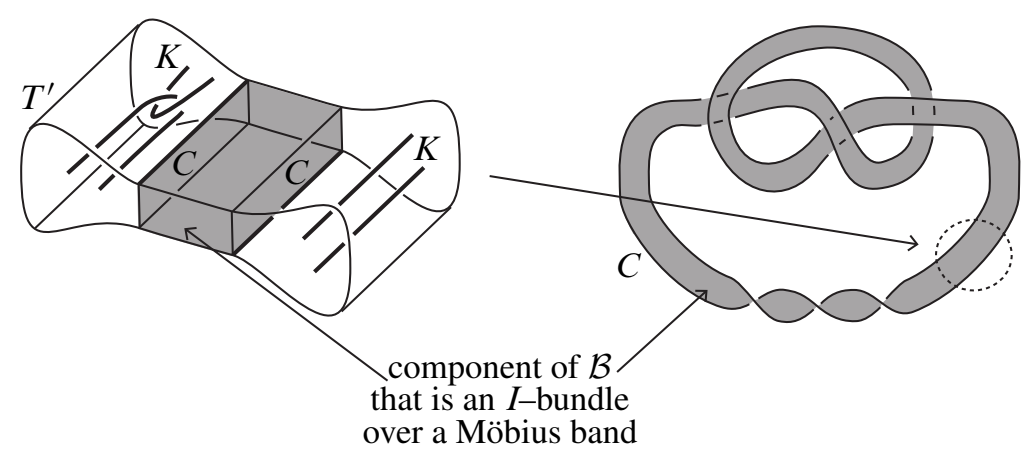

Figure 12

Let $C$ be one of these 2-cables. We now wish to find an upper bound for the crossing number of $C$. We will do this by finding a bound on the number of crossings in the diagram of $C$ that is obtained by projecting $C$ vertically onto the horizontal plane.

Now, $C$ has the structure of a cell complex, where each 1-cell is a component of the intersection between a handle of $\partial_{h} \mathcal{B}$ and a handle of $T$ that does not lie in $\partial_{h} \mathcal{B}$. Each such 1-cell either lies in the boundary of a 0 -handle of $\mathcal{H}_{X}$ or it lies in a 1-handle of $\mathcal{H}_{X}$ and respects its product structure. When we project $C$ vertically, the images of the latter 1-cells do not intersect each other or the image of any other 1-cell. Thus, the only way that crossings of the diagram of $C$ arise is from the 1-cells of $C$ that lie in $\mathcal{H}_{X}^{0}$. Each such 1-cell lies in the boundary of a normal disc of $T$ that does not lie in $\mathcal{B}$. There are at most 10 such normal discs, consisting of at most 2 squares and at most 8 triangles. Each square of $T$ has in its boundary 81 -cells, each of which is straight in the polyhedral structure on the 0 -handle of $\mathcal{H}_{X}$. Each triangle of $T$ has in its boundary 6 straight arcs. Thus, in each 0 -handle of $\mathcal{H}_{X}, C$ is composed of at most 64 straight arcs. We may perturb these arcs a little so that, when projected to the horizontal plane, the images of any two arcs intersect in at most one point. So, the resulting diagram of $C$ at most $64 \times 64 / 2$ crossings arising from each 0 -handle of $\mathcal{H}_{X}$. There are $(4 c(D)+2) 0$-handles of $X$, which is at most $(14 / 3) c(D)$. So, the number of crossings of $C$ is at most $9558 c(D)$.

Almost exactly the same argument gives that the modulus of the twisting of this 2-cable is at most $9558 c(D)$. We may consider a curve $C^{\prime}$ in $T$ parallel to $C$. Then, the twisting of the cable is half the linking number of $C$ and $C^{\prime}$. The modulus of this linking number is at most the number of crossings between $C$ and $C^{\prime}$ when they are vertically projected. So, we get the same upper bound of $9558 c(D)$ for the modulus of the twisting number.

The reason for bounding the crossing number of $C$ and the twisting of the 2-cable is that these are key quantities in the following theorem, which will be proved in Section 5. 
Theorem 5.1 Let $C$ be a knot that is a 2-cable of a knot $L^{\prime}$ with twisting number $t$. Then, $c\left(L^{\prime}\right) \leq 119024(c(C)+|t|)$.

Applying this result to our situation gives that $c\left(L^{\prime}\right) \leq 119024 \times 2 \times 9558 c(D)<$ $3 \times 10^{9} c(D)$, which is better than the required bound. This proves Theorems 1.1 and 1.4 in this case.

Case 2 No component of $\mathcal{B}$ is an $I$-bundle over a Möbius band.

Our aim is to construct a handle structure $\mathcal{H}_{V^{\prime}}$ for an isotopic copy $V^{\prime}$ of the solid torus $V$. The proof now divides according to whether the conditions of Theorem 3.10 are met by $\mathcal{H}_{Y^{\prime}}$, viewed as a handle structure for $\left(Y^{\prime}, T^{\prime}\right)$.

Case 2A There is no essential annulus properly embedded in $Y^{\prime}$ with boundary in $T^{\prime}$ and that lies entirely within the union of the parallelity handles of $\mathcal{H}_{Y^{\prime}}$.

By Theorem 3.10, we may assume that $\mathcal{B}$ is a collection of $I$-bundles over discs. We replace each component of $\mathcal{B}$ by a $2-$ handle, forming a handle structure $\mathcal{H}_{Y^{\prime}}^{\prime}$. This inherits an affine structure from $\mathcal{H}_{Y^{\prime}}$, since according to the definition, we do not need to identify 2 -handles with polyhedra.

The next step is to add a 2 -handle as a thickened meridian disc for $K$. This 2 -handle is attached along the exceptional 0 -handles and 1 -handles. Finally, a 3 -handle is added in the remainder of $N(K)$. The result is a handle structure $\mathcal{H}_{V^{\prime}}$ for $V^{\prime}=Y^{\prime} \cup N(K)$. We can give it an affine structure as follows. We simply declare that the polyhedra associated with each unexceptional 0 -handle and 1 -handle is the same as that in $\mathcal{H}_{Y^{\prime}}^{\prime}$. The exceptional 0 -handles and 1-handles must be modified slightly to ensure that the new 2-handles intersect each of these polyhedra in convex polygons. Thus, it is easy to arrange that the conditions in the definition of an affine handle structure are satisfied by $\mathcal{H}_{V^{\prime}}$.

Case 2B There is some properly embedded essential annulus $A$ in $Y^{\prime}$ with boundary in $T^{\prime}$, and that lies in the union of the parallelity handles of $\mathcal{H}_{Y^{\prime}}$.

Then $A$ is properly embedded in the solid torus $V^{\prime}=Y^{\prime} \cup N(K)$. Now, any properly embedded annulus in a solid torus $V^{\prime}$ satisfies at least one of the following:

(i) It is boundary parallel.

(ii) Both its boundary curves are meridians and the annulus forms a knotted tube joining these.

(iii) At least one of its boundary curves bounds a disc in $\partial V^{\prime}$. 


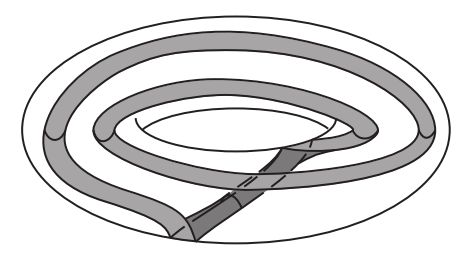

(i)

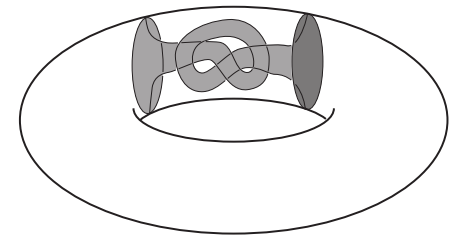

(ii)

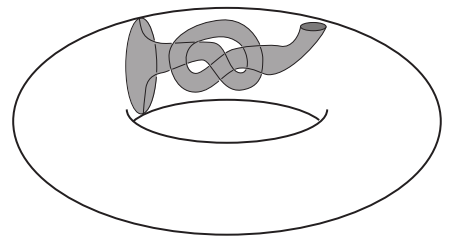

(iii)

Figure 13

We claim that (ii) and (iii) do not arise. For suppose that (ii) holds. Then $A$ cuts $V^{\prime}$ into two pieces, one of which is homeomorphic to the exterior of a non-trivial knot $K^{\prime}$, and the other of which is a solid torus $W$. Now, $K$ must lie in $W$ because the former piece lies within a 3-ball in $V^{\prime}$. Also, $K$ does not lie in a 3-ball in $W$, since $K$ would then lie in a 3-ball in $V^{\prime}$. In addition, $K$ is not a core curve of $W$, because this would imply that $K$ has $L^{\prime}$ as a connected summand, and we are assuming that this does not occur. Thus, $K$ is a non-trivial satellite of $L^{\prime} \sharp K^{\prime}$, which is contrary to our assumption about $L^{\prime}$. Now suppose that (iii) holds. Then the disc in $\partial V^{\prime}$ that is bounded by one of the components of $\partial A$ becomes a compression disc for $A$ in $Y^{\prime}$, which contradicts the assumption that $A$ is essential in $Y^{\prime}$. This proves the claim.

Thus, $A$ is boundary parallel in $V^{\prime}$. Since $A$ is essential in $Y^{\prime}, K$ must lie in the parallelity region $P$ between $A$ and a sub-annulus of $T^{\prime}$. We take $A$ to be innermost in $V^{\prime}$, in the sense that any other essential annulus properly embedded in $Y^{\prime}$ with boundary in $T^{\prime}$ and which lies within the union of the parallelity handles must lie in $P$. Then $A$ is a vertical boundary component of $\mathcal{B}$. Moreover, the component of $\mathcal{B}$ that is incident to $A$ lies in $P$. This is because this component of $\mathcal{B}$ cannot be an $I$-bundle over a Möbius band, and so it is an $I$-bundle over an annulus. So, if this component of $\mathcal{B}$ did not lie in $P$, this would contradict the fact that we have taken $A$ to be innermost in $V$.

Remove $P-A$ from $V^{\prime}$. (See Figure 14.) The resulting 3-manifold is an isotopic copy of $V^{\prime}$, which we still call $V^{\prime}$. It inherits an affine handle structure. The intersection $\mathcal{B} \cap V^{\prime}$ is a generalised parallelity bundle, consisting of $I$-bundles over discs. Replace each of these $I$-bundles with a 2 -handle, forming the required affine handle structure $\mathcal{H}_{V^{\prime}}$ for $V^{\prime}$. Its only parallelity handles are 2 -handles. 


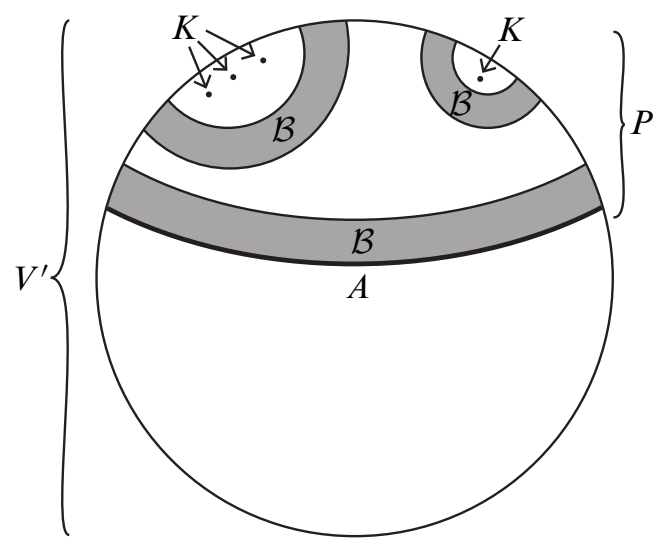

Figure 14

Thus, in each of Cases $2 \mathrm{~A}$ and 2B, we have created an isotopic copy $V^{\prime}$ of $V$, and it has an affine handle structure $\mathcal{H}_{V^{\prime}}$.

We claim that $\mathcal{H}_{V^{\prime}}$ satisfies the conditions of Theorem 1.3. Note that $\mathcal{H}_{X}$ satisfies these conditions, apart from the fact that $X$ is not a solid torus. Then $\mathcal{H}_{V^{\prime}}$ was obtained from $\mathcal{H}_{X}$ by the following operations:

(i) cutting along a closed properly embedded normal surface

(ii) removal of handles (but maintaining a handle structure)

(iii) replacing generalised parallelity bundles by 2 -handles

(iv) adding some handles (when filling in $N(K)$ in Case 2A)

It is clear that the conditions of Theorem 1.3 are preserved under (i), (ii) and (iii). The addition of handles in (iv) also does not violate the conditions of Theorem 1.3, because the 2-handles that are added are attached to the remnants of the exceptional 0 -handles and 1-handles. The exceptional 1-handles of $\mathcal{H}_{X}$ each intersect $\mathcal{H}_{X}^{2}$ in two components, and so the addition of a further 2-handle does not violate the hypotheses of Theorem 1.3. This proves the claim.

Each 0 -handle and 1 -handle of $\mathcal{H}_{V^{\prime}}$ is a handle of $\mathcal{H}_{Y}$. Thus, each 0 -handle of $\mathcal{H}_{V^{\prime}}$ lies in a 0 -handle of $\mathcal{H}_{X}$. Moreover, each 0 -handle of $\mathcal{H}_{X}$ contains at most six 0 -handles of $\mathcal{H}_{V^{\prime}}$. This is because each 0 -handle of $\mathcal{H}_{X}$ can support at most 5 types of triangles and squares of $T$ that are simultaneously disjoint. These therefore divide the handle into at most six 0 -handles that are not parallelity handles. The remaining 0 -handles of $\mathcal{H}_{Y}$ are parallelity 0 -handles, and are therefore removed in the construction of $\mathcal{H}_{V^{\prime}}$. 
We now apply Theorem 1.3 to deduce that $V^{\prime}$ has a core curve $C$ that lies in the 0 -handles and 1-handles of $\mathcal{H}_{V^{\prime}}$, that respects the product structure on the 1-handles, and that intersects each 0 -handle of $\mathcal{H}_{V^{\prime}}$ in at most 48 arcs. Moreover, these arcs are parallel to arcs $\alpha$ in the boundary of the 0 -handle, and each component of $\alpha$ intersects each face of the 0 -handle in at most 6 straight arcs. We calculated above that each 0 -handle of $\mathcal{H}_{X}$ gave rise to at most six non-parallelity 0 -handles of $\mathcal{H}_{Y}$ and hence at most six 0 -handles of $\mathcal{H}_{V^{\prime}}$. We also calculated that these 0 -handles of $\mathcal{H}_{Y}$ have at most 236 faces. Hence, the same is true of the 0 -handles of $\mathcal{H}_{V^{\prime}}$. (The exceptional 0 -handles do not exceed this bound.) So, within each 0 -handle of $\mathcal{H}_{X}, C$ consists of at most $6 \times 48 \times 236=67968$ straight arcs.

We now project $C$ vertically to form a diagram $D^{\prime}$ of $L^{\prime}$. Now, $C$ lies in the $0-$ handles and 1-handles of $\mathcal{H}_{X}$, and within the 1-handles, it respects their product structure. The interiors of the 0 -handles and the 1-handles have disjoint images under the projection map. So, the only place that crossings of $D^{\prime}$ can occur is within the images of the 0 -handles of $\mathcal{H}_{X}$. We have calculated that within each 0 -handle, $C$ consists of at most 67968 straight arcs. These give rise to at most $(67968)^{2}$ crossings. There are $(4 c(D)+2) 0$-handles of $X$, which is at most $(14 / 3) c(D)$. So, the number of crossings of $D^{\prime}$ is at most $(14 / 3)(67968)^{2} c(D)<3 \times 10^{10} c(D)$, as required.

Thus, in order to complete the proof of Theorems 1.1 and 1.4, all that remains for us to do is prove Theorem 5.1. This we do in the next section.

\section{The crossing number of 2 -cables}

In this section, we prove a version of the main theorem in a very special case, where the satellite $\operatorname{knot} K$ is a $2-$ cable.

Theorem 5.1 Let $K$ be a knot that is a 2-cable of a knot $L$ with twisting number $t$. Then, $c(L) \leq 119024(c(K)+|t|)$.

We may clearly assume that $L$ is a non-trivial knot, as otherwise, the statement of the theorem is empty.

Let $X$ be the exterior of $K$. Since $K$ is a 2-cable, it forms the boundary of an embedded Möbius band, the core of which is a copy of $L$. Let $F_{0}$ be the restriction of this Möbius band to $X$.

Let $D$ be a diagram for $K$ with minimal crossing number. We now modify $D$ by performing Type I Reidemeister moves, which introduce kinks. We perform enough of these moves so that the writhe of the new diagram $D^{\prime}$ is equal to twice the twisting of 
the 2-cable. Thus, $D^{\prime}$ has crossing number at most $2 c(K)+2|t|$. Using this diagram, give $X$ the affine handle structure described in Section 2, but without the introduction of the exceptional handles. We denote this by $\mathcal{H}_{X}$. Note that the number of 0 -handles of $\mathcal{H}_{X}$ is $4 c\left(D^{\prime}\right)$, which is at most $8(c(K)+|t|)$.

We may pick a simple closed curve on $\partial N(K)$ that winds once along $N(K)$ and that has blackboard framing with respect to $D^{\prime}$. We may arrange that it is standard in the handle structure and intersects each 0 -handle of $\mathcal{H}_{X}$ in at most two arcs. Moreover, it intersects each component of $\mathcal{H}_{X}^{0} \cap \partial X$ and $\mathcal{H}_{X}^{1} \cap \partial X$ in at most one arc. Since the writhe of $D^{\prime}$ is equal to twice the twisting of the 2-cable, this simple closed curve is ambient isotopic to $\partial F_{0}$. Thus, we may arrange $F_{0}$ so that its boundary is equal to this curve.

Because $L$ is non-trivial, $F_{0}$ is boundary-incompressible, and it is also incompressible. So, by Proposition 3.4, there is an ambient isotopy, supported in the interior of $X$, taking $F_{0}$ to a normal surface. This does not move the boundary of $F_{0}$.

Now let $F$ be a Möbius band properly embedded in $X$ such that:

(i) $F$ is normal.

(ii) $\partial F=\partial F_{0}$

(iii) $F$ is ambient isotopic to $F_{0}$.

Choose $F$ so that the pair $\left(\left|F \cap \mathcal{H}_{X}^{2}\right|,\left|F \cap \mathcal{H}_{X}^{0}\right|\right)$ is minimal among all properly embedded Möbius bands satisfying the above three conditions. Here, we are placing lexicographical ordering on such pairs. Thus, we first minimise $\left|F \cap \mathcal{H}_{X}^{2}\right|$, and then once this has smallest possible value, we minimise $\left|F \cap \mathcal{H}_{X}^{0}\right|$.

Let $N(F)$ be a thin regular neighbourhood of $F$ in $X$, which is an $I$-bundle over $F$, in which $F$ lies as a zero section. Let $\widetilde{F}$ be the associated $\partial I$-bundle over $F$, which is the annulus $\operatorname{cl}(\partial N(F)-\partial X)$.

Since $F$ is normal, it inherits a handle structure, where the $i$-handles of $F$ are the components of $\mathcal{H}_{X}^{i} \cap F$. Similarly, $\widetilde{F}$ inherits a handle structure.

Let $M$ be the result of cutting $X$ along $F$. Then $M$ inherits a handle structure $\mathcal{H}_{M}$. Note that $\widetilde{F}$ is a subsurface of $\partial M$ and its boundary is standard in $\mathcal{H}_{M}$. Thus, $\mathcal{H}_{M}$ is a handle structure for the pair $(M, \widetilde{F})$. Let $\mathcal{B}$ be its parallelity bundle.

Note that because $\partial F$ runs over each component of $\mathcal{H}_{X}^{0} \cap \partial X$ and $\mathcal{H}_{X}^{1} \cap \partial X$ at most once, no parallelity handle of $\mathcal{H}_{M}$ intersects $\partial \widetilde{F}$. Hence, $\partial_{h} \mathcal{B}$ lies in the interior of $\widetilde{F}$. Let $\widetilde{\Gamma}$ be the boundary of $\partial_{h} \mathcal{B}$, which is therefore a collection of simple closed curves in $\widetilde{F}$. We give $\widetilde{\Gamma}$ a cell structure, where each 1 -cell is a component of intersection 


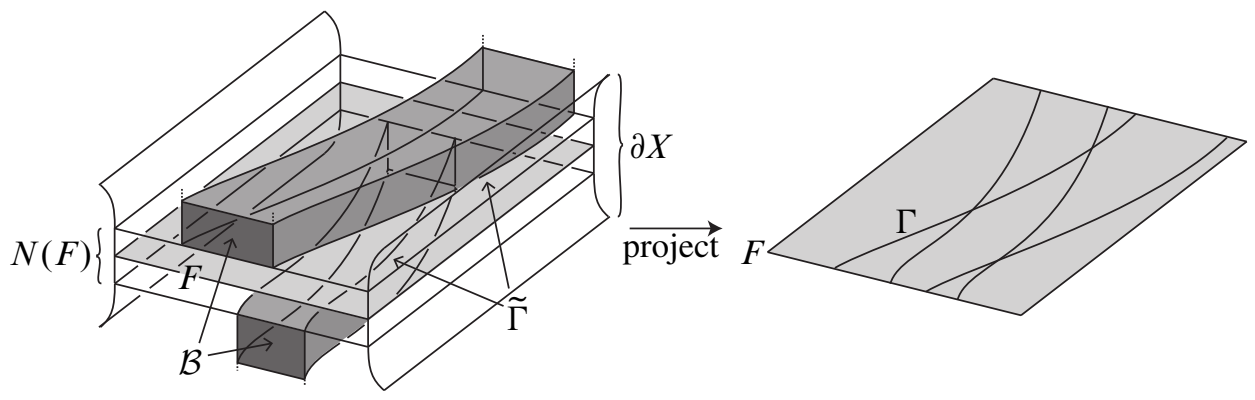

Figure 15

between adjacent handles of $\widetilde{F}$. Let $\Gamma$ be the image of $\widetilde{\Gamma}$ in $F$ under the bundle map $\widetilde{F} \rightarrow F$. Then $\Gamma$ is also a $1-$ complex. (See Figure 15 .)

Case $1 \Gamma$ contains a core curve $C$ of $F$ as a subcomplex.

Note that $\Gamma$ has controlled intersection with each handle of $\mathcal{H}_{X}$, in the following sense. Each 1-cell of $\Gamma$ lies in the image of a $1-$ cell of $\widetilde{\Gamma}$. This is a component of intersection between two handles of $\widetilde{F}$. One, $H_{1}$ say, lies in $\partial_{h} \mathcal{B}$, and the other, $H_{2}$, does not lie in $\partial_{h} \mathcal{B}$. We will now control the possibilities for $\mathrm{H}_{2}$ within each 0 -handle of $\mathcal{H}_{X}$. Now in each 0 -handle of $\mathcal{H}_{X}$, at most two normal discs of $F$ intersect $\partial X$. Suppose that there is such a disc $E$. Then each 1-cell of $E \cap \Gamma$ arises as a component of $E \cap \mathcal{F}^{0}$ or $E \cap \mathcal{F}^{1}$. We may arrange that each such 1-cell is straight in the affine structure on the 0 -handle. Now, $E$ runs over each component of $\mathcal{F}^{1}$ at most once, and so this gives rise to at most 61 -cells of $E \cap \Gamma$. Between these we have at most 5 components of $E \cap \mathcal{F}^{0}$ that miss $\partial X$. So, this gives at most 111 -cells of $\Gamma$ arising from $E$. The remaining normal discs of $F$ are squares and triangles, and so at most 10 of these can give a handle $H_{2}$ of $\widetilde{F}$ not in $\partial_{h} \mathcal{B}$. At most 2 of these are squares, and at most 8 are triangles. So, this gives at most $(2 \times 8)+(8 \times 6)=641$-cells of $\Gamma$. Again, we may arrange that each of these is straight. So, in each 0 -handle of $\mathcal{H}_{X}$, we have at most 86 straight 1 -cells of $\Gamma$. Projecting the core curve $C$, we obtain a diagram for $L$. The crossings of this diagram occur only in the projections of the 0 -handles of $\mathcal{H}_{X}$. There are at most $8(c(K)+|t|)$ such 0 -handles. So, we get a diagram for $L$ with at most $\frac{1}{2} \times(86 \times 85) \times 8(c(K)+|t|)=29240(c(K)+|t|)$ crossings, which is better than the required bound.

Case $2 \Gamma$ does not contain a core curve of $F$ as a subcomplex.

We claim that we may find a core curve $C$ of $F$ that avoids $\Gamma$. To see this, pick an ordering on the 1-cells of $\Gamma$, and remove an open regular neighbourhood of these 1 -cells from $F$ one at a time. At each stage, we examine the complementary regions. Initially, this is just a Möbius band. We will show that, at each stage, one complementary 
region is a Möbius band with a (possibly empty) collection of open discs removed. We call this a punctured Möbius band. Moreover, a core curve of this punctured Möbius band is also a core curve of $F$. Note this complementary region either contains all of $\partial F$ or is disjoint from $\partial F$ because $\Gamma$ is disjoint from $\partial F$. As each new 1-cell of $\Gamma$ is removed from $F$, there are three options. It may be completely disjoint from the previous cells, in which case this just punctures one of the complementary regions. It may have just one endpoint incident to the previous cells, in which case the complementary regions are unchanged up to ambient isotopy. The main case is where both endpoints of the 1-cell are incident to earlier cells. In this case, we cut a complementary region along a properly embedded $\operatorname{arc} \alpha$. We are only interested in the case where this region is the punctured Möbius band. If this joins different punctures, the result is still a punctured Möbius band. If $\alpha$ joins a puncture to the boundary of the punctured Möbius band, then cutting along $\alpha$ still results in a punctured Möbius band. If $\alpha$ joins the boundary to itself, the result is an arc properly embedded in the Möbius band. If the arc is essential, then we have a core curve of $F$ as a subcomplex of $\Gamma$, which is contrary to hypothesis. So, the arc is inessential, and there remains a complementary region that is a punctured Möbius band. If the arc joins a puncture to itself, the result is a simple closed curve embedded within the Möbius band. This is either inessential, in which case there remains a complementary region that is a punctured Möbius band, or it is essential, in which case this leads to a core curve of $F$ as a subcomplex of $\Gamma$, and again this is contrary to hypothesis. This proves the claim.

Let $C$ be this core curve. After an isotopy in the complement of $\Gamma$, we may assume that it intersects each handle of $F$ in at most one properly embedded arc. Let $M_{1}$ be the union of the $I$-fibres in $N(F)$ over $C$. This is a Möbius band. Let $\widetilde{C}$ be the boundary of $M_{1}$. Then $\widetilde{C}$ misses $\widetilde{\Gamma}$. It therefore misses $\partial_{h} \mathcal{B}$ or lies in the interior of $\partial_{h} \mathcal{B}$. The argument divides into these two cases.

Case 2A $\tilde{C}$ misses $\partial_{h} \mathcal{B}$.

Then $C$ lies in the surface $F_{-}$that is obtained from $F$ by removing the interior of the image of $\partial_{h} \mathcal{B}$ under the bundle map $\widetilde{F} \rightarrow F$. Note that whenever an $i$-handle of $\tilde{F}$ lies in $\partial_{h} \mathcal{B}$, so do all the $j$-handles with $j>i$ that are incident to it. Hence, $F_{-}$inherits a handle structure. We may therefore ensure that $C$ misses the 2-handles of $F_{-}$and respects the product structure on the 1 -handles. We may also isotope $C$ in $F_{-}$so that it intersects each handle of $F_{-}$in at most one properly embedded arc. Because $\widetilde{C}$ misses $\partial_{h} \mathcal{B}$, each handle of $F$ that intersects $C$ is disjoint from $\mathcal{B}$ on both sides. There can be at most 7 such normal discs of $F$ in each 0 -handle of $X$ : at most 4 triangles, at most one square and at most two further normal discs that intersect $\partial X$. As in Section 4, we may arrange that each triangle is made up 16 flat polygons and that the square is made up of 25 flat polygons. Similarly, the normal discs that intersect 
$\partial X$ consist of at most 84 polygons in total. We may ensure that $C$ intersects each of the polygons in at most one straight arc. So, in each 0 -handle of $\mathcal{H}_{X}, C$ is at most $(4 \times 16)+25+84=173$ straight arcs. Therefore, the projection of $C$ has at most $\frac{1}{2}(173 \times 172) \times 4 c\left(D^{\prime}\right) \leq 119024(c(K)+|t|)$ crossings, as required.

Case 2B $\widetilde{C}$ lies in $\partial_{h} \mathcal{B}$.

Our aim here is to reach a contradiction. Let $\mathcal{B}^{\prime}$ be the component of $\mathcal{B}$ that contains $\widetilde{C}$. Then $\partial_{h} \mathcal{B}^{\prime}$ is either connected or disconnected, depending of whether the base surface of the $I$-bundle $\mathcal{B}^{\prime}$ is non-orientable or orientable. We consider these two cases separately.

Case 2B(i) $\partial_{h} \mathcal{B}^{\prime}$ is connected.

Then the base surface of $\mathcal{B}^{\prime}$ is non-orientable, and therefore contains a properly embedded, orientation-reversing simple closed curve. The union of the fibres in $\mathcal{B}^{\prime}$ over this curve is a Möbius band $M_{2}$. Its boundary is a simple closed curve in the annulus $\tilde{F}$. This cannot bound a disc in $\widetilde{F}$, for then the union of $M_{2}$ with this disc would be an embedded projective plane in $S^{3}$, which is well known to be impossible. Thus, $\partial M_{2}$ is a core curve of $\widetilde{F}$ and therefore separates $\widetilde{F}$ into two annuli. Attach one of these annuli to $M_{2}$ to form a properly embedded Möbius band $M_{3}$ in $X$. The boundary curves of $M_{3}$ and $F$ are disjoint and therefore cobound an annulus in $\partial X$. Attach this annulus to $M_{3} \cup F$ to form an embedded Klein bottle in $S^{3}$. (See Figure 16.) This again is a contradiction.

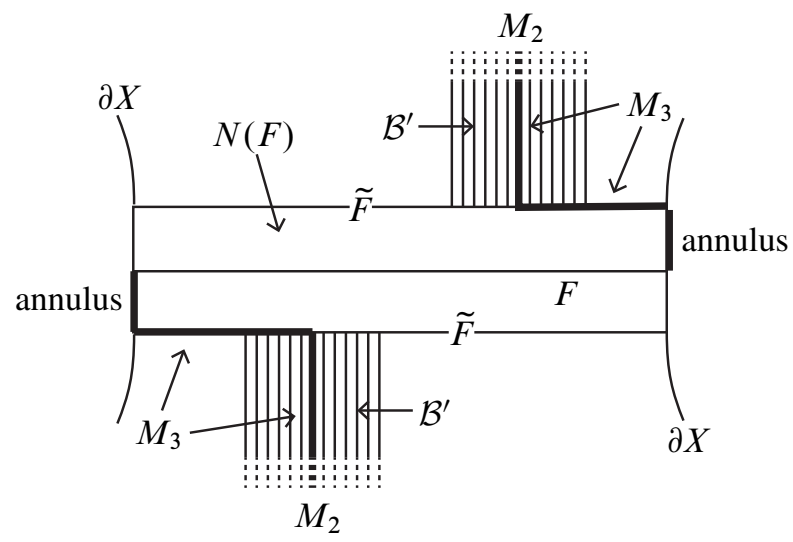

Figure 16

Case 2B(ii) $\partial_{h} \mathcal{B}^{\prime}$ is disconnected.

Then, $\mathcal{B}^{\prime}$ is a product $I$-bundle. Let $A_{1}$ be the union of the $I$-fibres in $\mathcal{B}^{\prime}$ that are incident to $\widetilde{C}$. This is an annulus. The boundary curve $\partial A_{1}-\widetilde{C}$ is a simple closed 
curve in $\tilde{F}$. It cannot bound a disc in $\tilde{F}$, for then the union of this disc with $A_{1} \cup M_{1}$ would be an embedded projective plane in $S^{3}$. So, $\partial A_{1}-\widetilde{C}$ divides $\widetilde{F}$ into two annuli. Let $A_{2}$ be the annulus that does not contain $\widetilde{C}$. Let $A_{3}$ be the sub-annulus of $\widetilde{F}$ lying between the two components of $\partial A_{1}$. (See Figure 17.)

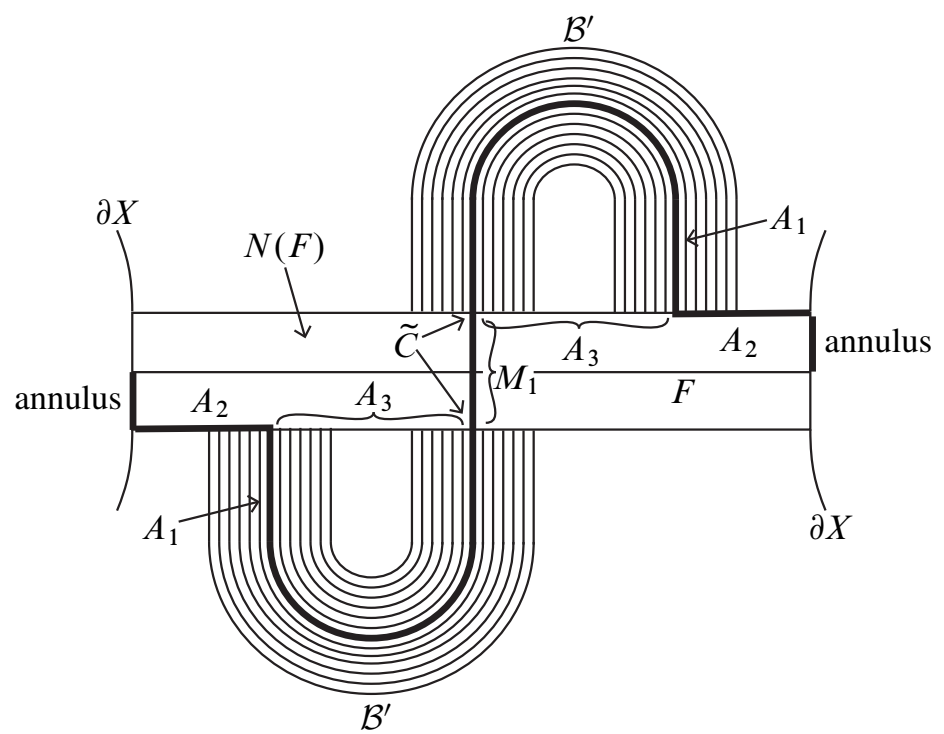

Figure 17

Note that $\widetilde{F}-\widetilde{C}$ consists of two annuli. The restriction of the bundle map $\widetilde{F} \rightarrow F$ to each of these annuli is an injection. In particular, the restriction of $\widetilde{F} \rightarrow F$ to $A_{2} \cup A_{3}-\tilde{C}$ is an injection.

We claim that $\partial A_{1}-\widetilde{C}$ intersects each handle of $\widetilde{F}$ in at most one properly embedded arc. Now, when a handle of $\widetilde{F}$ intersects $\widetilde{C}$, it does so in one properly embedded arc. Since $\widetilde{C}$ lies in $\mathcal{B}$, this handle of $\widetilde{F}$ runs parallel to another handle of $\widetilde{F}$, with the region between these two handles lying in $\mathcal{B}$. So, $\partial A_{1}-\widetilde{C}$ intersects this other handle of $\widetilde{F}$ in just one arc.

Thus, each handle of $\widetilde{F}$ is divided into at most two components by $\partial A_{1}-\widetilde{C}$ and at most one of these lies in $A_{2}$. So, each $i$-handle of $F$ gives rise to at most one component of $A_{2} \cap \mathcal{H}_{X}^{i}$. Hence, for $i=0,1$ and $2,\left|A_{2} \cap \mathcal{H}_{X}^{i}\right| \leq\left|F \cap \mathcal{H}_{X}^{i}\right|$.

We claim that this inequality is strict for $i=0$. Note that whenever an $i$-handle of $\widetilde{F}$ lies in $\mathcal{B}$, so does every $j$-handle adjacent to it, provided $j>i$. So, $\operatorname{cl}\left(\widetilde{F}-\partial_{h} \mathcal{B}\right)$ has a handle structure. In particular, each component of $\operatorname{cl}\left(\widetilde{F}-\partial_{h} \mathcal{B}\right)$ contains a 0 -handle of $\tilde{F}$. So, $A_{3}$ contains at least one 0 -handle of $\widetilde{F}$. Hence, $\left|A_{2} \cap \mathcal{H}_{X}^{0}\right|<\left|F \cap \mathcal{H}_{X}^{0}\right|$, as claimed. 
Let $F_{0}^{\prime}$ be the Möbius band $M_{1} \cup A_{1} \cup A_{2}$. This is properly embedded in $X$, and after a small isotopy, we may arrange that it has the same boundary as $F$. It has strictly fewer components of intersection with the 0 -handles of $X$ than $F$, and at most as many components of intersection with $\mathcal{H}_{X}^{2}$. We have verified this for $A_{2}$. But $M_{1}$ is composed of $I$-fibres in $N(F)$, and $A_{1}$ is composed of $I$-fibres in $\mathcal{B}$. Hence, adding these to $A_{2}$ does not increase the number of components of intersection with $\mathcal{H}_{X}^{0}$ or $\mathcal{H}_{X}^{2}$. Now, $F_{0}^{\prime}$ might not be a normal surface, but when we apply the usual normalisation procedure (leaving the boundary fixed), the complexity of the pair $\left(\left|F_{0}^{\prime} \cap \mathcal{H}_{X}^{2}\right|,\left|F_{0}^{\prime} \cap \mathcal{H}_{X}^{0}\right|\right)$ does not increase. Thus, we end with a normal Möbius band properly embedded in $X$, with the same boundary as $F$, but with smaller complexity. This is ambient isotopic to $F$ by the following result. Hence this gives a contradiction.

Lemma 5.2 Let $K$ be a knot in the 3-sphere. Let $F$ and $F_{0}$ be essential Möbius bands properly embedded in the exterior of $K$ with equal boundaries. Then $F$ and $F_{0}$ are ambient isotopic in the exterior of $K$.

Proof We make use of the JSJ decomposition of the exterior $X$ of $K$. The JSJ tori are a collection of disjoint properly embedded essential tori, with the property that any other properly embedded essential torus can be ambient isotoped off them. The JSJ tori divide $X$ into pieces that are Seifert fibred or atoroidal. Since $X$ is a knot exterior, the possibilities for the Seifert fibred pieces are very limited (see for example Budney's paper [1]). Each has base surface that is a planar surface. Moreover, if the Seifert fibered piece has any singular fibres, the base orbifold is either a disc with two singularities of coprime order, or an annulus with one singularity.

Let $W$ be a regular neighbourhood of $F \cup \partial N(K)$ in $X$. This is a Seifert fibre space. The boundary component $\partial W-\partial N(K)$ either bounds a solid torus with interior disjoint from $W$ or is essential in $X$. In both cases, it can be ambient isotoped off the JSJ tori, and then $W$ lies in a Seifert fibred piece $R$ of the JSJ decomposition. We can then arrange that the $W$ is a union of fibres in $R$, and that the Möbius band $F$ is also a union of fibres. Thus, $F$ contains a singular fibre of order 2 , and it projects to an embedded arc in the base orbifold of $R$. This arc runs from $\partial R$ to the order 2 singularity. Note that there is just one order 2 singularity in this orbifold, because if there is another, it has coprime order. Now, removing the singularities from the orbifold gives a pair of pants $P$, and there is, up to ambient isotopy, a unique properly embedded arc in $P$ joining any two boundary components.

Now let $W^{\prime}$ be a regular neighbourhood of $F_{0} \cup \partial N(K)$ in $X$. Then, $W^{\prime}$ also lies in $R$ after an ambient isotopy, and it too is a union of fibres in $R$, as is $F_{0}$. Thus, after removing the singular fibres, $F_{0}$ projects to an $\operatorname{arc}$ in $P$ that is isotopic to the previous one. Hence, $F_{0}$ is ambient isotopic to $F$. 
This completes the proof of Theorem 5.1, and hence Theorems 1.1 and 1.4.

\section{A related result}

Recall that a knot $\widetilde{L}$ is a cable of a knot $L$ if $\widetilde{L}$ is a simple closed curve on $\partial N(L)$ that does not bound a disc in $N(L)$.

Theorem 1.5 Let $K$ be a satellite knot with companion knot $L$. Suppose that $L$ is prime. Then, for some knot $\widetilde{L}$, which is either $L$ or a cable of $L$,

$$
c(K) \geq \frac{c(\tilde{L})}{152} .
$$

Proof Let $D$ be a diagram for $K$ with minimal crossing number. Let $X$ be the exterior of $K$. Give $X$ the handle structure described in Section 2, but without the introduction of the exceptional handles. Call this handle structure $\mathcal{H}_{X}^{\prime}$. We do not give it an affine structure, but instead, realise the handles as shown in Figure 2 and the left of Figure 5. Let $T$ be the torus $\partial N(L)$. This may be placed in normal form with respect to $\mathcal{H}_{X}^{\prime}$. Cutting $X$ along $T$ gives two 3 -manifolds, one of which is a copy of the exterior of $L$. Call this latter manifold $Z$, and let $\mathcal{H}_{Z}$ be the handle structure that it inherits. View this as a handle structure for the pair $(Z, T)$.

Apply as many annular simplifications to $\mathcal{H}_{Z}$ as possible, creating a handle structure $\mathcal{H}_{Z}^{\prime}$. According to Theorem $3.9, \mathcal{H}_{Z}^{\prime}$ has a generalised parallelity bundle $\mathcal{B}$ that contains every parallelity handle of $\mathcal{H}_{Z}^{\prime}$ and which has incompressible horizontal boundary $\partial_{h} \mathcal{B}$.

Now $\partial_{h} \mathcal{B}$ cannot be all of $\partial Z$, because $Z$ would then be an $I$-bundle over a torus or Klein bottle. Hence, $\partial_{h} \mathcal{B}$ is a (possibly empty) collection of discs and a (possibly empty) collection of annuli.

Claim If $\partial_{h} \mathcal{B}$ contains any annuli, then these do not have meridional slope.

Suppose that, on the contrary, these annuli have meridional slope. The vertical boundary of the corresponding components of $\mathcal{B}$ are then incompressible annuli properly embedded in $Z$, with meridional boundary. They cannot be essential, because $L$ would then be a composite knot. Hence, they are boundary parallel. Note that, in this case, no component of $\mathcal{B}$ can be an $I$-bundle over a Möbius band. For then $\mathcal{B}$ would contain a properly embedded Möbius band that is a union of $I$-fibres, and this could be capped off with discs to form an embedded projective plane in $\mathbb{R}^{3}$, which is known not to exist. Thus, the vertical boundary components of $\mathcal{B}$ with meridional boundary come in 
pairs that lie in the same component of $\mathcal{B}$. We may therefore pick one such annulus $A$, with the property that the component of $\mathcal{B}$ that it lies in is part of the parallelity region between $A$ and $\partial Z$. Removing this parallelity region (apart from $A$ itself) is therefore an annular simplification that can be made to $\mathcal{H}_{Z}^{\prime}$, which is contrary to hypothesis. This proves the claim.

Let $F$ be the surface $\partial Z-\operatorname{int}\left(\partial_{h} \mathcal{B}\right)$. This is either a collection of punctured annuli or a punctured torus, where the punctures arise from components of $\mathcal{B}$ that are $I$-bundles over discs. It inherits a handle structure. In the case where it is a collection of punctured annuli, we may pick a core curve $\alpha$ of one of these annuli, which misses the punctures, which runs only over the 0 -handles and 1 -handles, which respects the product structure on the 1-handles, and which intersects each handle in at most one arc. For we may start with a core curve $\alpha$ of one of the annuli, slide it off the punctures and the 2-handles, and then straighten it in the 1 -handles. If $\alpha$ intersects some handle in more than one $\operatorname{arc}$, then we may find an $\operatorname{arc} \beta$ in the handle, joining distinct arcs of $\alpha$ in that handle, and with interior disjoint from $\alpha$. One can then cut $\alpha$ at the two points of $\partial \beta$, remove one of the resulting arcs, and replace it by $\beta$. The result is still a core curve of the annulus, but which intersects fewer handles.

The other case is when $F$ is a punctured torus. This time we pick a curve $\alpha$ that avoids the punctures and the 2-handles, and that respects the product structure on the 1-handles, and that has longitudinal slope, say, on $\partial Z$. This time, it may not be possible to isotope $\alpha$ so that it runs over each handle in at most one arc. The modifications described above will not necessarily keep $\alpha$ as a longitude. However, they will not change its class in $H_{1}(\partial Z ; \mathbb{Z} / 2)$. So, we may still find a simple closed curve $\alpha$ on $F$ that runs over each handle in at most one arc, that misses the 2-handles and that respects the product structure on the 1-handles, that is essential in $\partial Z$ and that is not a meridian.

Let $\widetilde{L}$ be this curve $\alpha$. It is either isotopic to $L$ or is a cable of $L$.

We now have to be a little more precise about the position of $\tilde{L}$. We first arrange that each normal disc of $T$ sits within the handle of $\mathcal{H}_{X}^{\prime}$ that contains it as described in Section 6.5 of [6]. Then we arrange that, whenever $\widetilde{L}$ runs over one of these normal discs, then it does so as described in Section 6.5 of [6]. We then project $\tilde{L}$ vertically, forming a diagram $\widetilde{D}$, and we have to bound its crossing number. The details of the argument are identical to those of the author in Section 6.6 of [6]. In particular, the argument there gives that $c(\tilde{D}) \leq 152 c(D)$. This proves the theorem, because

$$
c(\tilde{L}) \leq c(\tilde{D}) \leq 152 c(D)=152 c(K) .
$$


We close with some final remarks about the nature of this proof. The arguments behind Theorems 1.1 and 1.5 are similar, but in the former case, we focused on $Y=N(L)-\operatorname{int}(N(K))$, whereas in the latter case, we used $Z=S^{3}-\operatorname{int}(N(L))$. Although the use of $Z$ leads to better a better constant, one loses track of where the meridian of $Z$ lies. It therefore seems very hard to avoid the possibility that the knot $\widetilde{L}$ we are considering might be a cable of $L$. Only by considering $Y$ and using Theorem 1.3 does it seem feasible to bypass this issue of cabling.

Nonetheless, Theorem 1.5 reduces the general problem of finding a lower bound on the crossing number of a satellite knot to the same problem for cables. It is conceivable that it can be used as part of an alternative proof of Theorem 1.1.

\section{References}

[1] R Budney, JSJ-decompositions of knot and link complements in $S^{3}$, Enseign. Math. 52 (2006) 319-359 MR2300613

[2] M H Freedman, Z-X He, Divergence-free fields: Energy and asymptotic crossing number, Ann. of Math. 134 (1991) 189-229 MR1114611

[3] J Hoste, M Thistlethwaite, J Weeks, The first 1,701,936 knots, Math. Intelligencer 20 (1998) 33-48 MR1646740

[4] R Kirby, Editor, Problems in low-dimensional topology, from: "Geometric topology", (W H Kazez, editor), AMS/IP Stud. Adv. Math. 2, Amer. Math. Soc. (1997) 35-473 MR1470751

[5] M Lackenby, Core curves of triangulated solid tori, to appear in Transactions of the AMS

[6] M Lackenby, The crossing number of composite knots, J. Topol. 2 (2009) 747-768 MR2574742

[7] S Matveev, Algorithmic topology and classification of 3-manifolds, Algorithms and Computation in Mathematics 9, Springer, Berlin (2003) MR1997069

Mathematical Institute, University of Oxford

Radcliffe Observatory Quarter, Woodstock Road, Oxford OX2 6GG, UK

lackenby@maths.ox.ac .uk

Received: 18 May 2012 Revised: 22 August 2013 
